Original Article

\title{
Pattern of Renal and Urinary Tract Diseases Among Children Admitted to A General Ward Over One Year
}

\author{
Magd A. Kotb ${ }^{1}$, Noha Adel Yassin'1, M. Amr Lotfi ${ }^{2}$, Shaimaa Sayed ${ }^{*}$ \\ 1 Department of Pediatrics, Faculty of Medicine, Cairo University, Egypt; magdkotb@kasralainy.edu.eg, \\ noha.adel20@yahoo.com \\ 2 Department of Urology, Faculty of Medicine, Cairo University, Egypt; profamrlotfi@kasralainy.edu.eg \\ * Correspondence: shaimaasayed@kasralainy.edu.eg \\ Received: 21/11/2021; Accepted: 20/12/2021; Published online: 27/12/2021.
}

\begin{abstract}
:
Background: Children with renal and urinary tract diseases may require admission in general pediatric wards.

Aim of the work: To identify the pattern of renal and urinary tract diseases among children admitted to general pediatric ward and their associated morbidities and mortality.

Methods: Retrospective analysis of files of children with confirmed diagnosis of renal and urinary tract diseases who were admitted to a general pediatric ward, at Children Hospital, Cairo University.

Results: During 12 months, 142 patients with renal or urinary tract disease were admitted to our pediatric general ward. Of them $97(68.3 \%)$ were boys and $45(31.7 \%)$ were girls. Their mean age was $5.1 \pm 3.89$ years. Of them 121 (85.2\%) had isolated renal disease and 21 (14.8\%) had renal affection as a part of systemic diseases. The most common diseases among those with isolated renal disease, were glomerular diseases (62 patients, $51.2 \%$ ), followed by congenital anomalies of the kidney and urinary tract (33 patients, 27.3\%). Different types of infection were the main cause for admission among 78 (54.9\%) children. Thirty four patients (23.9\%) underwent renal replacement therapy, and 26 patients suffered from poor outcome.

Conclusion: Pediatricians should have the knowledge of the different presentations of renal disease in children and be trained for timely referral to the pediatric nephrologists and/or urologist. Renal diseases should be suspected in those with diseases of other systems. Infections are the commonest renal indication of admission in the general pediatric ward.
\end{abstract}

Level of Evidence of Study: IIB (1).

Keywords: Pediatric urinary tract disease; renal diseases; hospital stay; renal replacement therapy; urinary tract infection.

Abbreviations: AKI: acute kidney injury; CAKUT: congenital anomalies of the kidney and urinary tract; CKD: Chronic kidney disease; CVC: central venous catheter; HUS: hemolytic uremic syndrome; PUV: posterior urethral valve; UT: urinary tract; UTI: urinary tract infection.

\section{Introduction}

Renal diseases are a health problem in pediatric population (2). Urinary tract (UT) diseases encompass diseases of kidneys, ureters, urinary bladder and urethra. The pediatric general ward provides services limited by scope of expertise of team of professional health workers, the available equipment and operating setting. Though demanding training is necessary to provide the required skills to become proficient pediatric nephrologist (3) or pediatric urologist (4), the general pediatrician is also qualified to handle diagnosis, management and timely referral of the child with urinary tract disease (5). General pediatric wards admit children with a spectrum of UT diseases, as urinary tract infections (UTI) (6), acute post infectious glomerulonephritis, vasculitis as Henoch-Schönlein, hemolytic uremic syndrome, renal failure, urolithiasis and a vast array of diseases (7).

Renal disease in pediatric population may be asymptomatic and detected accidentally during a routine physical examination (8). Unexplained symptoms like fever, pains, anemia, 
gastrointestinal symptoms, abdominal mass, edema, hypertension, and metabolic acidosis maybe early signs of renal disease. Chronic kidney disease (CKD) may present by anorexia, lassitude, anemia, growth failure and/or hypertension (9).

The aim of this work was to identify the pattern of renal diseases among children admitted to general department at Children Hospital, Cairo University Pediatric Hospitals which is a tertiary hospital that serves children from different governorates and their associated morbidities and mortality.

\section{Subjects and Methods}

This retrospective study was conducted on data and medical records of patients admitted to a single General Pediatric Ward of the Children Hospital, Cairo University Pediatric Hospitals, Egypt from October 2020 to September 2021. The study applied the ethical principles for medical research involving human subjects of The Declaration of Helsinki (10).

All medical data of pediatric patients aged from 30 days to 14 years, who were admitted to a single General Pediatric Ward, in Children Hospital, Cairo University Pediatric Hospitals who were admitted with known, or were newly diagnosed as having a renal disease during hospitalization were included. Collected information included: the age, gender, primary renal disease (if known), indication for hospital admission, duration of hospital admission, the presence of hypertension, the presence of clinical or laboratory evidence of infection, performance of invasive procedure or therapeutic intervention, need for renal replacement therapy and outcome including morbidity or mortality. Long hospital stay was considered when the patient spends 10 days or more at the hospital (11). The presence of hypertension was included in data collection which is defined as high blood pressure values $\geq 95$ th percentile based on age, sex, and height on three separate visits (12). Uncontrolled hypertension was considered when the values of blood pressure were above $\geq 95$ th percentile based on age, sex, and height, in spite of receiving antihypertensive medications. Hypertensive encephalopathy is defined as acute brain dysfunction such as severe headache, changes in consciousness, seizure, and retinal hemorrhage induced by sustained severe hypertension (13).

Accepted outcome was considered when the patient was discharged from hospital and properly treated without developing any complications during his hospital stay; as proper treatment of infections (in case of presence of infections), proper control of his blood pressure (in case of presence of hypertension), resolution of generalized edema (in case of nephrotic syndrome), improvement of renal functions (in case of presence of renal impairment).

Poor outcome was considered when the patient developed any complications during the hospital stay despite receiving adequate proper treatment.

\section{Statistical Analysis}

Data were coded and entered using the statistical package for the Social Sciences. (SPSS Statistics for Windows, Version 17.0. Chicago: SPSS Inc). Data were summarized using mean and standard deviation for quantitative variables; and frequencies for categorical variables. Comparisons between groups were done using analysis of variance (ANOVA) with multiple comparisons post hoc test in normally distributed quantitative variables while non-parametric Kruskal-Wallis test and Mann-Whitney test were used for non-normally distributed quantitative variables (14). For comparing categorical data, Chi square (x2) test was performed. Fisher's exact test was used instead when the expected frequency is less than 5 (15). Correlation coefficients and tests of significance were employed. P-values $<0.05$ were considered as statistically significant.

\section{Results}

The current study included 142 children who were admitted at a single Pediatric General Ward of the existent six general wards of the Cairo University Pediatric Hospital over a period of one year from October 2020 to September 2021. Among the 142 children, 71 children were newly diagnosed and the other 71 children were known to have renal or UT disease. The total number of children admitted at the same department at the same period was 999 patients. Children with renal disease represented $14.2 \%$ of the total number of the admitted children in 
the same ward during the same year. Of them 45 patients (31.7\%) were females, and 97 patients (68.3\%) were males. The mean age at admission was 5 years and 43 days \pm 3 years and 10 months (median age of the patients was 4.5 years, range was $1-168$ months) (Table 1). The mean total hospital stay duration was $11.8 \pm 9.3$ days.

Table 1: Age at admission and gender distribution of the studied cohort

\begin{tabular}{|c|c|c|c|c|c|}
\hline & Number & Percentage & $\begin{array}{c}\text { Mean age } \\
\text { in years }\end{array}$ & SD & $\begin{array}{c}\mathbf{P} \\
\text { value }\end{array}$ \\
\hline \multicolumn{6}{|c|}{ All studied cohort } \\
\hline Males & 97 & 68.3 & 4.36 & 3.5 & \multirow[t]{2}{*}{0.002} \\
\hline Females & 45 & 31.7 & 6.5 & 4.11 & \\
\hline Total & 142 & 100 & 5.108 & 3.83 & \\
\hline \multicolumn{6}{|c|}{ Those with known renal disease } \\
\hline Males & 49 & 69 & 4.9 & 3.84 & \multirow[t]{2}{*}{0.038} \\
\hline Females & 22 & 31 & 7 & 3.59 & \\
\hline Total & 71 & 50 & 5.75 & 4.72 & \\
\hline \multicolumn{6}{|c|}{ Those newly diagnosed with renal disease during this hospital admission } \\
\hline Males & 48 & 67.6 & 4 & 3.83 & \multirow[t]{2}{*}{0.038} \\
\hline Females & 23 & 32.39 & 6.02 & 4.5 & \\
\hline Total & 71 & 50 & 4.65 & 3.83 & \\
\hline
\end{tabular}

SD: standard deviation, p-value: probability value

\section{Underlying renal disease among the studied children}

Only 71 patients $(50 \%)$ of those admitted were known to have renal or UT disease. The other 71 patients $(50 \%)$ were diagnosed as having renal or UT disease at the hospital during the study period. Among those admitted 21 (14.8\%) patients suffered renal disease associated with other system disease and $121(85.2 \%)$ patients had isolated renal disease. Among the patients who had isolated renal disease $62(51.2 \%)$ had glomerular diseases; 57 had different types of nephrotic syndrome (Figure 1), 4 had acute post streptococcal glomerulonephritis and one had Alport syndrome. Thirty three (27.2\%) patients had congenital anomalies of the kidney and urinary tract (CAKUT), 17 patients had vesicoureteral reflux, 10 patients had posterior urethral valve (PUV), 4 patients had pelvi-ureteric junction obstruction and 2 patients had neurogenic bladder. Eleven (9.1\%) patients had unknown etiology of the renal disease, they presented by end stage renal disease and had bilateral atrophic kidneys. Eight (6.7\%) patients had renal tubular disorder; 4 had renal tubular acidosis, 2 had Bartter's syndrome and 2 had autosomal recessive polycystic kidney disease. Seven (5.8\%) patients had urolithiasis. Twenty one patients had systemic diseases that had renal affection; 8 had hemolytic uremic syndrome (HUS), 11 patients had acute kidney injury (AKI) secondary to sepsis, nephrotoxic medications or dehydration and 2 patients had lupus nephritis secondary to systemic lupus erythematosus.

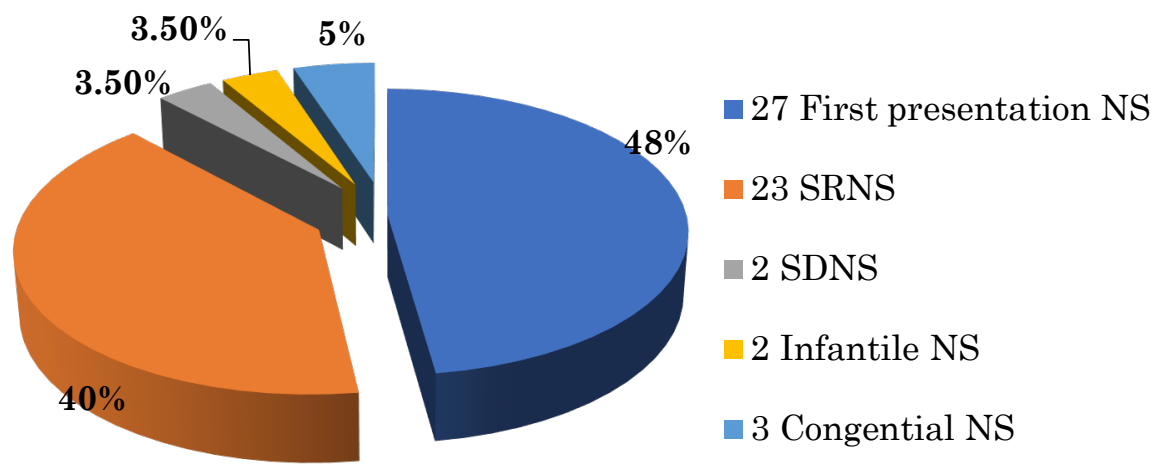

Figure 1: Types of nephrotic syndrome encountered among our studied cohort NS: nephrotic syndrome, SRND: steroid resistant nephrotic syndrome, SDNS: steroid dependent nephrotic syndrome. 


\section{Indication for hospital admission}

Sixty-two (43.66\%) children presented by acute presentations. 80 (56.3\%) patients presented by acute condition on top of CKD. Some patients had more than one cause for admission. The most common cause for admission was different types of infections that represent $55 \%$ (78 patients). 31 patients suffered from had UTI, 5 patients suffered from infective endocarditis, 11 chest infections, 19 patients suffered from gastroenteritis, 2 patients suffered from cellulites and 10 patients suffered from sepsis. These patients had clinical evidence (as fever) and/ or laboratory evidence of infection (as positive c reactive protein, leukocytosis and positive urine or blood cultures). Correlations between infection and different studied are shown in Table 2.

Table 2: Correlations between infection and different studied variables

\begin{tabular}{lcc}
\hline & $\mathbf{R}$ & $\mathbf{P}$ value \\
\hline RRT & 0.2062 & 0.0138 \\
\hline Acute presentations & 0.382 & 0.00001 \\
\hline Invasive intervention & 0.380 & 0.00001 \\
\hline Chronicity of renal disease & 0.426 & 0.00001 \\
\hline Gender & 0.069 & 0.4145 \\
\hline Age & 0.0197 & 0.8224 \\
\hline Underlying disease & 0.0524 & 0.5357 \\
\hline Hypertension & -0.1249 & 0.1414 \\
\hline
\end{tabular}

RRT: renal replacement therapy.

Forty (28.1\%) patients had hypertension, of them10 were admitted by uncontrolled hypertension and 2 of them had hypertensive encephalopathy. Twenty-one patients ( $15 \%$ of the total number of the study) were admitted due to acute onset of renal impairment secondary to systemic diseases, of them 8 had HUS, 11 patients had AKI secondary to sepsis, nephrotoxic medications, dehydration or massive hemolysis secondary to hemolytic anemia and 2 patients had lupus nephritis. All the 57 patients who suffered from different types of nephrotic syndrome had generalized edema. Some of them were admitted to receive albumin transfusion in addition to other indications for admission as different types of infections.

\section{Duration of hospital admission}

Median length of hospital stay for our studied cohort was 10 days with wide range from 1-60 days (mean $+/-\mathrm{SD}=11.8+/-9.3$ days). 76 patients (53.5\%) had long length hospital stay (equal or more than 10 days).

\section{Invasive procedures or therapeutic interventions}

Seventy children underwent an invasive procedure or therapeutic intervention. Twenty patients had central venous catheter (CVC) insertion; that were inserted at the Nephrology Unit, Children Hospital, Cairo University Pediatric Hospitals, and were used for renal replacement therapy (RRT). Two patients had upper endoscope examination; as they suffered from persistent vomiting and it was performed to diagnose the cause of persistent vomiting after failure of medical management. Eleven patients had performed renal biopsy for diagnosis of primary renal disease. One patient had chest tube insertion as he had empyema. Forty patients had urinary catheter insertion; to relive lower urinary tract obstruction or as a part of another invasive procedure as during performance of peritoneal dialysis.

Three patients had received plasma transfusion and/ or plasma exchange as a part of management of atypical HUS. Thirty-four (23.9\%) patients needed renal replacement therapy; 17 patients had acute indication of RRT and 17 patients continued on chronic RRT). Five patients had cystoscope examination to diagnose and treat lower UT obstruction as PUV or for insertion of ureteric stent.

One patient had unilateral nephrectomy as a part of management of unresolving persistent pyelonephritis in non-functioning kidney. One patient had vesicostomy to treat lower UT obstruction.

\section{Outcome (morbidity or mortality)}

Hypertension complicated the course in 40 (28.1\%) children. Hypertension correlated with older age $(r=0.2040, p=0.014)$, longer hospital stay $(r=0.2431, p=0.0035)$, need for RRT $(r=$ $0.1744, \mathrm{p}=0.03)$, but not to gender $(\mathrm{r}=0.078, \mathrm{p}=0.356)$ or prior knowledge of renal disease $(\mathrm{r}=$ 
$0.1252, \mathrm{p}=0.137)$. So, hypertensive patients may need longer hospital stay to control their blood pressure.

Twenty-six patients suffered from poor outcome in the form of development of complications or mortality during their hospital stay; 8 developed hospital acquired infections in the form of CVC related infections, diarrhea, chest infection, wound infection or cellulites. 8 needed ICU admission. 3 developed deep venous thrombosis. 4 patients suffered from bleeding; one had retinal hemorrhage, two developed hematemesis and one had urethral bleeding secondary to urethral injury. 2 patients suffered from convulsions secondary to hypertensive encephalopathy. One patient developed arrhythmia secondary to digitalis toxicity. Three patients died; 2 developed septic shock and one died after development of arrhythmia secondary to digitalis toxicity. Renal replacement therapy was associated with better outcome $(r=0.1847, p=0.027)$. There was no correlation between patients' age and poor outcome $(\mathrm{r}=-0.0477, \mathrm{p}=0.572)$, type of renal disease $(r=-0.0746, p=0.381)$, hospital stay duration $(r=-0.0942, p=0.265)$ and performance of invasive procedure $(\mathrm{r}=0.0078, \mathrm{p}=0.926)$.

\section{Discussion}

Primary renal disease is an important predictor of CKD progression in children $(2,16)$. Pediatricians should have the knowledge of the different presentations of renal disease in children, and a high index of suspicion to diagnose disease that could be asymptomatic. Typically change of color of urine, or abnormal urine test examination as proteinuria, fluid overload, and electrolyte disturbances are the commonest causes for referral to the pediatric nephrologists (17). Referral to a pediatric nephrologist is done once diagnosis of nephrotic syndrome, acute or chronic glomerulonephritis, UTI, hypertension, and CKD is made. Early diagnosis and treatment of renal disease could lead to the timely treatment and prevention of CKD in pediatric population.

The current study included 142 patients, of them 45 (31.7\%) were females, and 97 (68.3\%) were males. The more prevalence of affection of males might be related to the primary renal disease. The most common renal disease in the current study was nephrotic syndrome, which affected 57 patients. It might be more common in males than females. Also, 33 patients had CAKUT; which is strongly associated with male sex (18).

There was age difference between males and females; females were significantly older than males in the study group and subgroups. We could not find a clear explanation about age difference between males and females in the literature. But, in general late presentation is a global problem among children with chronic kidney disease, with higher incidence in low/middleincome countries. Children presenting late are older and more likely to have non-congenital kidney disease as in females (19).

In the current study, infections were the commonest complications or associations with renal or urinary tract disease in hospitalized sick children in the general ward. The infections encountered in our studied cohort were not only related to urinary tract, but also to other systems infections as infective endocarditis, chest infections, gastroenteritis, cellulites and sepsis. Infections are abundant as children with renal disease have compromised immunity (20), invasive interventions (8) and virulence of organisms (21). All these factors are known to increase susceptibility to infection $(8,20,21)$. Infections in our studied cohort did not correlate with patient age or gender, but correlated with acute presentations ( $p$-value $=0.00001)$ and chronicity of the renal disease ( $p$-value $=0.00001)$. This suggests that patients who had infections presented with acute presentation on top a chronic renal disease. Also, infection correlated with RRT (pvalue $=0.0138$ ), and invasive intervention ( $p$-value $=0.0001)$. $R R T$ was a risk factor for infection; that could be due to presence of invasive device as inserted CVC used during hemodialysis or inserted urinary catheter during performance of peritoneal dialysis or after insertion of ureteric stent. It is essential that the laboratory services and imaging studies affiliated to general ward serving children with potential and known renal disease be equipped for tests essential to diagnose and follow up a child with renal disease and infections. Regarding the underlying renal disease, we found that 33 (23\%) patients had CAKUT and all of them suffered from UTI. This was in agreement with that CAKUT are a common cause of pediatric CKD and should be suspected in children with recurrent UTI (18). Underlying molecular genetic factors are 
responsible for syndromic and non-syndromic CAKUT (22). CAKUT represented a quarter of our admitted cases, which seems to be related to the common practice of consanguineous marriages Egypt reaching $35.5 \%$ (23).

CAKUT can be diagnosed prenatally (24), and it causes serious morbidity and mortality. Prenatal diagnosis seems a priority to reduce CAKUT burden in Egypt.

Also, we found that $11(7.7 \%)$ patients presented by accidently discovered end stage renal disease and had unknown etiology of the renal disease. Renal disease in pediatric population may be asymptomatic and detected accidentally during a routine physical examination (9). Unexplained symptoms like fever, pains, anemia, gastrointestinal symptoms, abdominal mass, edema, hypertension, and metabolic acidosis may be early signs of renal disease. CKD may present by anorexia, lassitude, anemia, growth failure and/or hypertension (25).

Hypertension was an important complication of renal disease among our studied cohort. Hypertension is a disease that should be diagnosed and managed promptly given its potential of life threatening complications (27). General ward physicians should be proficient in diagnosis and management of hypertension in children. Hypertension is a modifiable cause for the progression of pediatric CKD (21); as the kidney is a major site for target organ damage of hypertension and hypertension could lead to progressive nephron damage $(27,28)$.

Renal replacement therapy remains the gold standard for severe acute renal failure, end stage renal disease and other indications (29). It was an important lifesaving procedure $(\mathrm{p}=0.027)$ for those children with indications for RRT in renal or urinary tract disease hospitalized in the general ward. RRT needs a specialized hospital setting (30), and not the regular general ward setting (31). Hence, RRT outcomes in the general ward are related to the proximity of specialized units that provide RRT and other interventions indicated for renal or urinary tract disease. Our studied cohort who received RRT were managed at our affiliated Pediatric Nephrology Unit, Children Hospital, Faculty of Medicine Cairo University. RRT requires highly trained professionals and not only the equipped setting (32).

\section{Conclusion}

Renal diseases are responsible for $14 \%$ of hospital admissions in the general ward. More than half of them presented by acute condition on top of CKD. Infections and hypertension are common renal indications for hospitalization in the general pediatric ward. A third of the children needed RRT. Renal diseases should be suspected in those with diseases of other systems. Pediatricians should be made aware of importance timely referral to the pediatric nephrologists and/or urologist. Tertiary pediatric hospital should have available facilities that help in diagnose of renal diseases and liaison with nephrology and urology units that cooperate to provide the best service for management of renal diseases of hospitalized children pediatric.

\section{Author Contributions}

All authors shared in conceptualization, supervising, data curation, data analysis, writing original draft, data interpretation, writing original draft, supervising and revising. All authors reviewed the final manuscript. All authors have read and agreed to the published version of the manuscript.

\section{FUNDING}

Authors declare there was no extramural funding provided for this study.

\section{CONFLICT OF INTEREST}

The authors declare no conflict of interest in connection with the study.

\section{References}

1. S. Tenny, M. Varacallo, Evidence Based Medicine. (StatPearls Publishing; Treasure Island (FL), 2020; https://www.ncbi.nlm.nih.gov/books/NBK470182/).

2. Alan W. Partin, Craig A. Peters, Louis R. Kavoussi, Roger R. Dmochowski, Alan J. Wein, Campbell Walsh Wein Urology (Elsevier, 12 th., 2020; https://www.elsevier.ca/ca/product.jsp?isbn=9780323546423).

3. American Society of Nephrology, Training and Certification Nephrology Boards- American Board of Internal Medicine (ABIM), (available at https://www.asn-online.org/training/fellows/certification). 
4. K. V. Satish Kumar, S. N. Oak, Making of a pediatric urologist. Indian J. Urol. IJU J. Urol. Soc. India. 23, 384-386 (2007).

5. Mayo Clinic, Academic General Pediatrician, (available at https:/apply.mayoclinic.org/TGnewUI/Search/home/HomeWithPreLoad?PageType=JobDetails\&partner $\mathrm{id}=25309$ \&siteid $=5280 \&$ jobid=2442134\&sourcecode=\%7Bvendor\%7D\#jobDetails=2442134_5280).

6. J. K. Leyenaar, S. L. Ralston, M.-S. Shieh, P. S. Pekow, R. Mangione-Smith, P. K. Lindenauer, Epidemiology of pediatric hospitalizations at general hospitals and freestanding children's hospitals in the United States. J. Hosp. Med. 11, 743-749 (2016).

7. S. J. Cunningham, P. Venkataraman, B. Goilav, in Clinical Manual of Emergency Pediatrics, E. Crain, J. Gershel, Eds. (Cambridge University Press, Cambridge, ed. 5, 2010; https://www.cambridge.org/core/product/identifier/9780511919268\%23c73687-23-1/type/book_part), pp. 644-667.

8. A. H. S. Cheung, L. M. F. Wong, SURGICAL INFECTIONS IN PATIENTS WITH CHRONIC RENAL FAILURE. Infect. Dis. Clin. North Am. 15, 775-796 (2001).

9. A. J. Barakat, Presentation of the Child with Renal Disease and Guidelines for Referral to the Pediatric Nephrologist. Int. J. Pediatr. 2012, 1-5 (2012).

10. World Medical Association, WMA Declaration of Helsinki- Ethical Principles for Medical Research Involving Human Subjects (2013), (available at https://www.wma.net/policies-post/wma-declaration-ofhelsinki-ethical-principles-for-medical-research-involving-human-subjects/2013/).

11. J. M. Gold, M. Hall, S. S. Shah, J. Thomson, A. Subramony, S. Mahant, V. Mittal, K. M. Wilson, R. Morse, G. M. Mussman, P. Hametz, A. Montalbano, K. Parikh, S. Ishman, M. O’Neill, J. G. Berry, Long length of hospital stay in children with medical complexity: LOS in Children With Medical Complexity. J. Hosp. Med. 11, 750-756 (2016).

12. National Institutes of Health, National Heart, Lung, and Blood Institute, The Fourth Report On The Diagnosis, Evaluation, and Treatment of High Blood Pressure in Children and Adolescents (2021), (available at https://www.nhlbi.nih.gov/sites/default/files/media/docs/hbp_ped.pdf).

13. A. Anitha, K. Babu, A 10-year study: Renal outcomes in patients with accelerated hypertension and renal dysfunction. Indian J. Nephrol. 30, 409 (2020).

14. A. Hart, Mann-Whitney test is not just a test of medians: differences in spread can be important. BMJ. 323, 391-393 (2001).

15. H.-Y. Kim, Statistical notes for clinical researchers: Chi-squared test and Fisher's exact test. Restor. Dent. Endod. 42, 152-155 (2017).

16. G. He, C. Li, X. Zhong, F. Wang, H. Wang, Y. Shi, L. Gan, J. Ding, Risk Factors for Progression of Chronic Kidney Disease With Glomerular Etiology in Hospitalized Children. Front. Pediatr. 9, 752717 (2021).

17. B. Viteri, J. Reid-Adam, Hematuria and Proteinuria in Children. Pediatr. Rev. 39, 573-587 (2018).

18. Z. Li, Y. Chen, L. Qiu, D. Chen, C. Hu, J. Xu, X. Zhang, Prevalence, types, and malformations in congenital anomalies of the kidney and urinary tract in newborns: a retrospective hospital-based study. Ital. J. Pediatr. 45, 50 (2019).

19. L. Plumb, E. J. Boother, F. J. Caskey, M. D. Sinha, Y. Ben-Shlomo, The incidence of and risk factors for late presentation of childhood chronic kidney disease: A systematic review and meta-analysis. PLOS ONE. 15, e0244709 (2020).

20. L. Jiang, P. Wen, in Chronic Kidney Disease, J. Yang, W. He, Eds. (Springer Singapore, Singapore, 2020; http://link.springer.com/10.1007/978-981-32-9131-7_12), pp. 153-159.

21. A. L. Flores-Mireles, J. N. Walker, M. Caparon, S. J. Hultgren, Urinary tract infections: epidemiology, mechanisms of infection and treatment options. Nat. Rev. Microbiol. 13, 269-284 (2015).

22. V. Capone, W. Morello, F. Taroni, G. Montini, Genetics of Congenital Anomalies of the Kidney and Urinary Tract: The Current State of Play. Int. J. Mol. Sci. 18, 796 (2017).

23. R. M. Shawky, M. Y. El-Awady, S. M. Elsayed, G. E. Hamadan, Consanguineous matings among Egyptian population. Egypt. J. Med. Hum. Genet. 12, 157-163 (2011).

24. A. N. Talati, C. M. Webster, N. L. Vora, Prenatal genetic considerations of congenital anomalies of the kidney and urinary tract (CAKUT). Prenat. Diagn. 39, 679-692 (2019).

25. K. Mola, D. Shimelis, PATTERN AND OUTCOME OF RENAL DISEASES IN HOSPITALIZED CHILDREN IN TIKUR ANBESSA SPECIALIZED TEACHING HOSPITAL, ADDIS ABABA, ETHIOPIA. Ethiop. Med. J. 54, 117-123 (2016).

26. S. Siddiqui, R. Malatesta-Muncher, Hypertension in Children and Adolescents: A Review of Recent Guidelines. Pediatr. Ann. 49 (2020), doi:10.3928/19382359-20200513-01.

27. N. Kamath, A. Iyengar, N. George, V. A. Luyckx, Risk Factors and Rate of Progression of CKD in Children. Kidney Int. Rep. 4, 1472-1477 (2019).

28. K. A. Griffin, Hypertensive Kidney Injury and the Progression of Chronic Kidney Disease. Hypertension. 70, 687-694 (2017).

29. F. I. Fadel, M. A. Kotb, M. A. Abdel Mawla, R. M. Hasanin, A. M. Salem, M. G. Fathallah, K. S. Amr, H. A. Ahmed, D. M. Salah, Primary hyperoxaluria type 1 in children: Clinical classification, renal 
replacement therapy, and outcome in a single centre experience. Ther. Apher. Dial., 1744-9987.13666 (2021).

30. A. R. Ahmed, A. Obilana, D. Lappin, Renal Replacement Therapy in the Critical Care Setting. Crit. Care Res. Pract. 2019, 1-11 (2019).

31. O. I. Odetunde, H. U. Okafor, S. N. Uwaezuoke, B. U. Ezeonwu, O. M. Ukoha, Renal Replacement Therapy in Children in the Developing World: Challenges and Outcome in a Tertiary Hospital in Southeast Nigeria. Sci. World J. 2014, 1-6 (2014).

32. H. Przybyl, J. Evans, L. Haley, J. Bisek, E. Beck, Training and Maintaining: Developing a Successful and Dynamic Continuous Renal Replacement Therapy Program. AACN Adv. Crit. Care. 28, 41-50 (2017).

CC O O 2021 submitted by the authors. Open access publication under the
terms and conditions of the Creative Commons Attribution (CC- BY-
NC- ND) license. (https://creativecommons.org/licenses/by-nc-nd/2.0/). 